\title{
Dynamics of neopentane in dense argon gases
}

\author{
H. E. Smorenburg, R. M. Crevecoeur, L. A. de Graaf, and I. M. de Schepper* \\ Interfacultair Reactor Instituut, Delft University of Technology, 2629 JB Delft, The Netherlands
}

(Received 11 July 1996)

\begin{abstract}
By means of inelastic neutron scattering we measure the dynamic structure factors $S(k, \omega)$ of three dense mixtures of neopentane and ${ }^{40} \mathrm{Ar}$ (invisible for neutrons) at $323 \mathrm{~K}$ for wave numbers $4<k<12 \mathrm{~nm}^{-1}$ and partial neopentane number densities $n_{\text {neo }}=0.4,2.7$, and $3 \mathrm{~nm}^{-3}$. For the first two samples, with deuterated neopentane $\mathrm{C}\left(\mathrm{CD}_{3}\right)_{4}, S(k, \omega)$ can be described by one Lorentzian in frequency $\omega$. For $k<5 \mathrm{~nm}^{-1}$ the halfwidth at half maximum is $\omega_{H}(k)=D_{c} k^{2}$, with $D_{c}=21$ and $13 \times 10^{-9} \mathrm{~m}^{2} \mathrm{~s}^{-1}$, the collective translational diffusion coefficients for neopentane at $n_{\text {neo }}=0.4$ and $2.7 \mathrm{~nm}^{-3}$, respectively. For $k>6 \mathrm{~nm}^{-1}$ and $n_{\text {neo }}=2.7$ $\mathrm{nm}^{-3}, \omega_{H}(k)$ shows a behavior reminiscent of "de Gennes narrowing." For $n_{\text {neo }}=3 \mathrm{~nm}^{-3}$, with $\mathrm{C}\left(\mathrm{CH}_{3}\right)_{4}$, the (incoherent) $S(k, \omega)$ can be described with two Lorentzians, due to translational and rotational motions of single neopentane molecules, yielding the self-diffusion coefficient $D_{s}=9 \times 10^{-9} \mathrm{~m}^{2} \mathrm{~s}^{-1}$ and the rotational diffusion coefficient $D_{r}=0.8 \pm 0.3 \mathrm{ps}^{-1}$. The results are consistent with kinetic theory calculations. [S1063-651X(96)04412-1]
\end{abstract}

PACS number(s): 66.10.Cb, 61.12.-q, 05.20.Dd

\section{INTRODUCTION}

The behavior of the dynamic structure factor $S(k, \omega)$ of dense noble gas fluid mixtures, as observed in inelastic neutron scattering experiments, has been successfully explained on the basis of the revised Enskog kinetic theory (RET) for hard sphere mixtures [1-4]. To do so one replaces the motion of the large (heavy) and small (light) particles in the real fluid by that of equivalent hard spheres with diameter $\sigma_{1}$ and $\sigma_{2}$, respectively. The RET does not yield the continuum Stokes-Einstein diffusion coefficient of one heavy particle with diameter $\sigma_{1}$ (the solute) dissolved in a solvent of (infinitesimally) light and small particles $\left(\sigma_{2} \rightarrow 0\right)$ [5]. Therefore the theory is restricted to diameter ratios $\sigma_{1} / \sigma_{2}$ not too different from 1 , and gradually loses its validity for increasing $\sigma_{1} / \sigma_{2}$ (i.e., for colloidal suspensions). It has been shown before that the RET gives a good description of the dynamics of fluid mixtures up to diameter ratios $\sigma_{1} / \sigma_{2}=1.4$ in a large range of concentrations of the solute $[3,4,6]$. Recently we have shown that, at very low concentrations of the solute $\left(\mathrm{C}_{60}\right)$ in the solvent $\left(\mathrm{CS}_{2}\right)$, the RET and the continuum Stokes-Einstein descriptions both are relevant at the diameter ratio $\sigma_{1} / \sigma_{2}=2.2$, indicating the existence of a region where both approaches overlap [7]. The validity of the RET for substantially higher concentrations of the solute in a solvent with diameter ratios $\sigma_{1} / \sigma_{2}>1.4$ has not been studied so far.

In this paper we examine the validity of the RET at a diameter ratio $\sigma_{1} / \sigma_{2}=1.7$, for low and high concentrations of the solute. In particular, we consider three mixtures of neopentane [2,2-dimethylpropane, $\mathrm{C}\left(\mathrm{CH}_{3}\right)_{4}$ or $\left.\mathrm{C}\left(\mathrm{CD}_{3}\right)_{4}\right]$ and ${ }^{40} \mathrm{Ar}$ with number densities of neopentane $n_{\text {neo }}=0.4,2.7$ [both $\left.\mathrm{C}\left(\mathrm{CD}_{3}\right)_{4}\right]$, and $3 \mathrm{~nm}^{-3}\left[\mathrm{C}\left(\mathrm{CH}_{3}\right)_{4}\right]$, respectively. We use neopentane and $\mathrm{Ar}$, because neopentane is (almost perfectly) spherical with effective hard sphere diameter $\sigma_{\text {neo }}=0.57 \mathrm{~nm}$ $[8,9]$, which is 1.7 times larger than that of $\operatorname{Ar}\left(\sigma_{\mathrm{Ar}}=0.335\right.$ $\mathrm{nm}[4]$ ), and thus in the $\sigma_{1} / \sigma_{2}$ region of our interest. Furthermore, neopentane and $\mathrm{Ar}$ are fully miscible for $p>252$ bars

\footnotetext{
*Author to whom all correspondence should be addressed.
}

at $T=323 \mathrm{~K}$ [9], which makes it possible to reach high concentrations of neopentane. Finally, by H/D isotope substitution in neopentane and the use of the ${ }^{40} \mathrm{Ar}$ isotope it is possible to study the incoherent and coherent dynamic structure factors of neopentane alone, since ${ }^{40} \mathrm{Ar}$ is virtually "invisible" for neutrons. We measure with the high resolution inelastic neutron scattering IRIS spectrometer of ISIS (Rutherford Appleton Laboratory, United Kingdom) the dynamic structure factors of these samples for wave numbers $4<k$ $<12 \mathrm{~nm}^{-1}$ and frequencies $-0.7<\omega<2.0 \mathrm{ps}^{-1}$. We find that the coherent dynamic structure factor $S(k, \omega)$, obtained for $n_{\text {neo }}=0.4$ and $2.7 \mathrm{~nm}^{-3}$, can be described by one Lorentzian in $\omega$, with half-width $\omega_{H}(k)$, which is due to the collective translational motions of neopentane molecules in Ar. For $k<5 \mathrm{~nm}^{-1}$ the translational motion is diffusive, i.e., $\omega_{H}(k)=D_{c} k^{2}$ with $D_{c}=21 \times 10^{-9} \mathrm{~m}^{2} \mathrm{~s}^{-1}\left(n_{\text {neo }}=0.4 \mathrm{~nm}^{-3}\right)$, and $D_{c}=13 \times 10^{-9} \mathrm{~m}^{2} \mathrm{~s}^{-1}\left(n_{\text {neo }}=2.7 \mathrm{~nm}^{-3}\right)$, the collective diffusion coefficients of neopentane. At higher wave numbers $k>8 \mathrm{~nm}^{-1}$ and $n_{\text {neo }}=0.4 \mathrm{~nm}^{-3}, \omega_{H}(k)$ shows a crossover to a linear behavior of $\omega_{H}(k)$ in $k$ approaching ideal gas free streaming. For $n_{\text {neo }}=2.7 \mathrm{~nm}^{-3}, \omega_{H}(k)$ shows a plateau at wave numbers $8<k<12 \mathrm{~nm}^{-1}$ close to the main peak in the static structure factor $S(k)$, reminiscent of "de Gennes narrowing" in simple fluids and dense colloidal suspensions. The half-widths $\omega_{H}(k)$ for both concentrations are in good agreement with those calculated with the RET.

The incoherent dynamic structure factor $S(k, \omega)$ for $n_{\text {neo }}=3 \mathrm{~nm}^{-3}$ can be described by two Lorentzians, the narrower one due to the translational motion of single neopentane molecules alone, and the second (broader) one due to a combination of translational and rotational motions of single neopentane molecules. We find a translational self-diffusion coefficient $D_{s}=9 \times 10^{-9} \mathrm{~m}^{2} \mathrm{~s}^{-1}$ and a rotational diffusion coefficient $D_{r}=0.8 \pm 0.3 \mathrm{ps}^{-1}$ for neopentane in Ar.

This paper is organized as follows. In Sec. II we describe the preparation of the samples, the neutron spectrometer, the data collection, and show typical inelastic neutron spectra $I(k, \omega)$ from which $S(k, \omega)$ is determined. We summarize Sears's "partial wave" expansion method [10] to describe the contributions of the translational and rotational motion in 
TABLE I. Sample properties: sample components, sample number, pressure $p$, number density $n_{\text {neo }}$ of neopentane and $n_{\mathrm{Ar}}$ of Ar, the effective hard sphere diameters $\sigma_{\text {neo }}$ of neopentane and $\sigma_{\mathrm{Ar}}$ of Ar, the reduced densities $n_{\text {neo }}^{*}$ and $n_{\mathrm{Ar}}^{*}$, macroscopic scattering cross section $\Sigma$, and diameter $d$ of the sample container. Uncertainties are given in parentheses.

\begin{tabular}{|c|c|c|c|c|c|c|c|c|c|c|}
\hline Sample & No. & $\begin{array}{c}p \\
\text { (bar) }\end{array}$ & $\begin{array}{c}n_{\text {neo }} \\
\left(\mathrm{nm}^{-3}\right)\end{array}$ & $\begin{array}{c}n_{\mathrm{Ar}} \\
\left(\mathrm{nm}^{-3}\right)\end{array}$ & $\begin{array}{l}\sigma_{\text {neo }} \\
(\mathrm{nm})\end{array}$ & $\begin{array}{c}\sigma_{\mathrm{Ar}} \\
(\mathrm{nm})\end{array}$ & $n_{\text {neo }}^{*}$ & $n_{\mathrm{Ar}}^{*}$ & $\frac{\Sigma}{\left(\mathrm{cm}^{-1}\right)}$ & $\begin{array}{c}d \\
(\mathrm{~cm})\end{array}$ \\
\hline $\begin{array}{c}\mathrm{C}\left(\mathrm{CD}_{3}\right)_{4} \\
{ }^{40} \mathrm{Ar}\end{array}$ & I & 300 & $\begin{array}{l}0.385 \\
(0.02)\end{array}$ & $\begin{array}{c}6.0 \\
(0.2)\end{array}$ & $\begin{array}{c}0.57 \\
(0.02)\end{array}$ & $\begin{array}{l}0.335 \\
(0.01)\end{array}$ & $\begin{array}{c}0.07 \\
(0.01)\end{array}$ & $\begin{array}{c}0.23 \\
(0.02)\end{array}$ & 0.1 & 2.8 \\
\hline $\begin{array}{c}\mathrm{C}\left(\mathrm{CD}_{3}\right)_{4} \\
{ }^{40} \mathrm{Ar}\end{array}$ & II & 300 & $\begin{array}{c}2.7 \\
(0.1)\end{array}$ & $\begin{array}{l}4.0 \\
(0.3)\end{array}$ & $\begin{array}{c}0.57 \\
(0.02)\end{array}$ & $\begin{array}{l}0.335 \\
(0.01)\end{array}$ & $\begin{array}{c}0.50 \\
(0.08)\end{array}$ & $\begin{array}{c}0.15 \\
(0.02)\end{array}$ & 0.5 & 0.18 \\
\hline $\begin{array}{c}\mathrm{C}\left(\mathrm{CH}_{3}\right)_{4} \\
{ }^{40} \mathrm{Ar}\end{array}$ & III & 630 & $\begin{array}{c}2.8 \\
(0.5)\end{array}$ & $\begin{array}{c}5.2 \\
(0.5)\end{array}$ & $\begin{array}{c}0.57 \\
(0.02)\end{array}$ & $\begin{array}{l}0.335 \\
(0.01)\end{array}$ & $\begin{array}{c}0.5 \\
(0.1)\end{array}$ & $\begin{array}{c}0.20 \\
(0.03)\end{array}$ & 3 & 0.075 \\
\hline
\end{tabular}

molecular liquids in Sec. III. In Sec. IV we apply the partial wave expansion method to the present samples and show the results. We end with a discussion in Sec. V.

\section{EXPERIMENT}

We describe the sample preparation in Sec. II A, and the neutron spectrometer and data collection in Sec. II B. We will show typical inelastic neutron spectra $I(k, \omega)$ for the three samples at the end of this section.

\section{A. Samples}

The samples were composed of deuterated neopentane $\mathrm{C}\left(\mathrm{CD}_{3}\right)_{4}$ (Cambridge Isotope Laboratories, $98 \%$ pure), hydrogenated $\mathrm{C}\left(\mathrm{CH}_{3}\right)_{4}$ (Phillips Petroleum Co., 99.97\% pure), and ${ }^{40} \mathrm{Ar}$ (Matheson Co., $99.95 \%$ pure). We consider three mixtures at $T=323 \mathrm{~K}$ : I, $\mathrm{C}\left(\mathrm{CD}_{3}\right)_{4}$ in ${ }^{40} \mathrm{Ar}(300$ bars, $\left.n_{\text {neo }}=0.4 \mathrm{~nm}^{-3}\right)$; II, $\mathrm{C}\left(\mathrm{CD}_{3}\right)_{4}$ in ${ }^{40} \mathrm{Ar}\left(300\right.$ bars, $n_{\text {neo }}=2.7$ $\left.\mathrm{nm}^{-3}\right)$; III, $\mathrm{C}\left(\mathrm{CH}_{3}\right)_{4}$ in ${ }^{40} \mathrm{Ar}\left(630\right.$ bars, $\left.n_{\text {neo }}=3 \mathrm{~nm}^{-3}\right)$. We will refer to these samples as samples I, II, and III, respectively. Their properties are summarized in Table I.

For each sample we use a different sample container to deal with the very different macroscopic scattering cross sections $\Sigma$ of the samples (see Table I). The sample container for sample I is a 7075 aluminum alloy single cylinder with inner diameter $28 \mathrm{~mm}$, and wall thickness $1.6 \mathrm{~mm}$. The containers for samples II and III are made of 5052 aluminum alloy capillaries that are bent back and forth into a plane of 19 and 38 parallel tubes, respectively. They are mounted in an aluminum frame covered with cadmium to avoid scattering from the frame. The capillary for sample II has an inner diameter of $1.8 \mathrm{~mm}$, and wall thickness of $0.4 \mathrm{~mm}$. The capillary for sample III has an inner diameter of $0.75 \mathrm{~mm}$, and wall thickness of $0.25 \mathrm{~mm}$. A detailed description of this container is given in [11].

\section{B. Spectrometer and data collection}

The experiments are performed on the spectrometer IRIS at the pulsed neutron source ISIS (Rutherford Appleton Laboratory, UK). IRIS is a time of flight inverted-geometry spectrometer with a frequency resolution $\Delta \omega=0.025 \mathrm{ps}^{-1}$ (full width at half maximum) using the (002) reflection of pyrolytic graphite analyzers in nearly backscattering geometry (analyzed wavelength $\lambda=0.667 \mathrm{~nm}[7,12]$. The energy transfer $\hbar \omega$ and momentum transfer $\hbar \mathbf{k}$ transferred to the sample by scattering of a neutron from an initial state with wave vector $\mathbf{k}_{0}$ (frequency $\omega_{0}$ ) to a final state with wave vector $\mathbf{k}_{1}=\mathbf{k}_{0}+\mathbf{k}$ (frequency $\omega_{1}$ ), is given by

$$
\begin{gathered}
\omega=\omega_{0}-\omega_{1}, \\
k=k_{1}\left[2+\omega / \omega_{1}-2 \cos (\phi) \sqrt{1+\omega / \omega_{1}}\right]^{1 / 2},
\end{gathered}
$$

where the frequency of the incoming neutrons $\omega_{0}=\omega_{0}(\tau)$ is directly determined by their time of flight $\tau, k_{1}=9.42 \mathrm{~nm}^{-1}$ and $\omega_{1}=2.79 \mathrm{ps}^{-1}$ are the wave number and frequency of the outgoing analyzed neutrons (wavelength $\lambda_{1}=0.667 \mathrm{~nm}$ ), $k=|\mathbf{k}|$, and $\phi$ is the scattering angle between $\mathbf{k}_{0}$ and $\mathbf{k}_{1}$. We determine the neutron intensities $I(\phi, \omega)$ as a function of scattering angle $\phi$ and frequency transfer $\omega$ for each sample in $12 \mathrm{~h}$. The data are collected by 30 detectors at angles $26^{\circ}<\phi<103^{\circ}$. At constant $\omega$ values, the spectra $I(\phi, \omega)$ are interpolated to spectra $I(k, \omega)$ at constant wave number $k$. The interpolated spectra $I(k, \omega)$ at wave numbers $k=4.5$, 6.3, 8.1, 9.9, and $11.7 \mathrm{~nm}^{-1}$ are shown in Fig. 1 for sample I, and in Fig. 2 for sample II. The intensities are not absolutely normalized, but for each sample the intensities $I(k, \omega)$ at different $k$ and $\omega$ values can be compared with one another. In Fig. 3 we plot $I(k, \omega)$ at wave numbers $k=4.5,6.3$, and $8.1 \mathrm{~nm}^{-1}$ for sample III. At higher wave numbers $k>8$ $\mathrm{nm}^{-1}$, the spectra $I(k, \omega)$ become too broad to observe any $\omega$ dependence. In the data analysis the resolution of the spectrometer $\left(\Delta \omega=0.025 \mathrm{ps}^{-1}\right)$ is irrelevant, since it is much narrower than all the spectra $I(k, \omega)$. Thus we obtain from $I(k, \omega)$ the experimental dynamic structure factors $S_{\text {expt }}(k, \omega)=I(k, \omega) / A$ of the samples where the absolute normalization factor $A$ will be discussed below.

\section{THEORY}

Here we discuss the theory for the experimental incoherent and coherent dynamic structure factors $S_{\text {expt }}(k, \omega)$ of the present neopentane $-{ }^{40} \mathrm{Ar}$ samples. We ignore the scattering from ${ }^{40} \mathrm{Ar}$ with respect to that from $\mathrm{C}\left(\mathrm{CD}_{3}\right)_{4}$ and $\mathrm{C}\left(\mathrm{CH}_{3}\right)_{4}$ (a numerical estimate of the ${ }^{40} \mathrm{Ar}$ scattering will be given at the end). Thus we can use Sears's "partial wave" expansion method [10] that determines the contributions of the translational and rotational motion in single component molecular liquids which we specialize to (nearly) spherical molecules. We consider $N$ identical molecules $j=1, \ldots, N$ each with $n$ nuclei $\nu=1, \ldots, n . S_{\text {expt }}(k, \omega)$ is then given by 


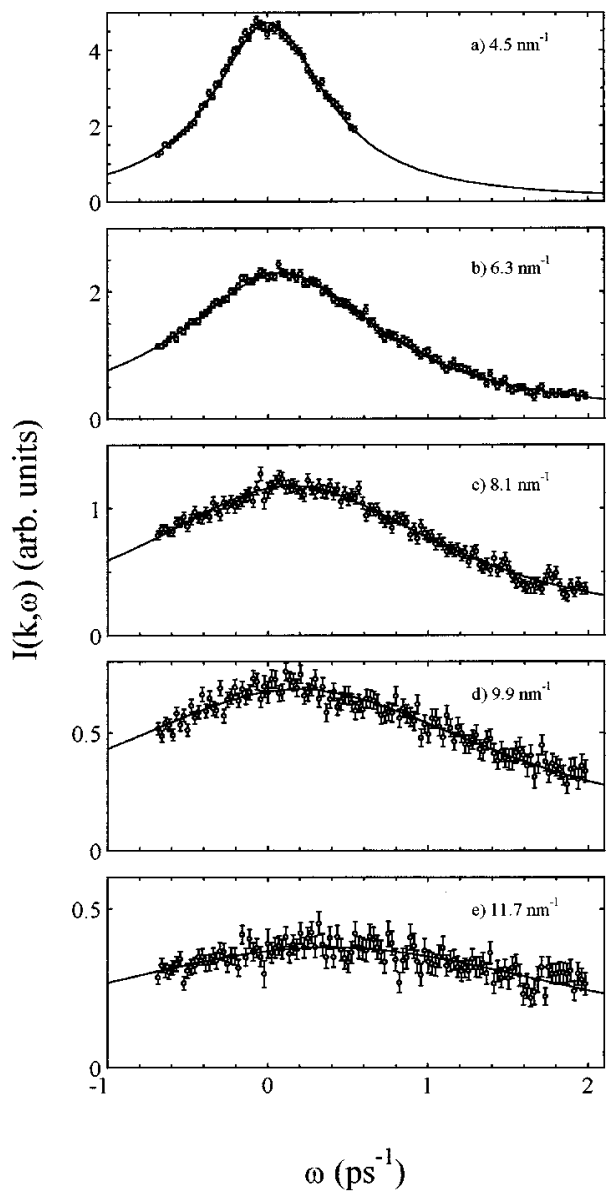

FIG. 1. Neutron intensities $I(k, \omega)$ as functions of frequency $\omega$ at wave numbers $k=4.5$ (a), 6.3 (b), 8.1 (c), 9.9 (d), and $11.7 \mathrm{~nm}^{-1}$ (e) of neopentane $\left[\mathrm{C}\left(\mathrm{CD}_{3}\right)_{4}\right]$ (number density $n_{\text {neo }}=0.4 \mathrm{~nm}^{-3}$ ) in ${ }^{40} \mathrm{Ar}$ at $323 \mathrm{~K}$ and 300 bars (sample I) $(\mathrm{O})$. The curves show the best one-Lorentzian fits $A S_{\text {expt }}(k, \omega)$, with $A$ the absolute normalization factor, and $S_{\text {expt }}(k, \omega)$ given in Eq. (14).

$$
S_{\text {expt }}(k, \omega)=\frac{1}{2 \pi} \int_{-\infty}^{\infty} d t \exp (-i \omega t) \sigma(\mathbf{k}, t)
$$

where

$$
\begin{aligned}
\sigma(\mathbf{k}, t)= & \frac{1}{N} \sum_{j, j^{\prime}=1}^{N} \sum_{\nu, \nu^{\prime}=1}^{n}\left\{b_{\mathrm{coh}}^{\nu} b_{\mathrm{coh}}^{\nu^{\prime}}+b_{\mathrm{inc}}^{\nu^{2}} \delta_{j j^{\prime}} \delta_{\nu \nu^{\prime}}\right\} \\
& \times\left\langle\exp \left\{-i \mathbf{k} \cdot \mathbf{R}_{j \nu}(0)\right\} \exp \left\{i \mathbf{k} \cdot \mathbf{R}_{j^{\prime} \nu^{\prime}}(t)\right\}\right\rangle,
\end{aligned}
$$

$\mathbf{R}_{j \nu}(t)$ is the position at time $t$ of nucleus $\nu=1, \ldots, n$ in molecule $j=1, \ldots, N$, and $b_{\text {coh }}^{\nu}$ and $b_{\text {inc }}^{\nu}$ are the bound coherent and incoherent scattering lengths for this nucleus. The brackets denote an average over an equilibrium canonical ensemble of all molecules. For (nearly) spherical molecules one may use the weak hindering approximation: one neglects statistical correlations between (i) the rotational and translational motion of one molecule and (ii) the rotational motion of different molecules. In particular Sears [10] has shown that the weak hindering approximation is applicable to molecular liquids where hydrogen bonding is absent, like for neopentane fluids in the present case. Then one can separate the center-of-mass motion from the rotational motion. The



FIG. 2. Neutron intensities $I(k, \omega)$ as functions of frequency $\omega$ at wave numbers $k=4.5$ (a), 6.3 (b), 8.1 (c), 9.9 (d), and $11.7 \mathrm{~nm}^{-1}$ (e) of neopentane $\left[\mathrm{C}\left(\mathrm{CD}_{3}\right)_{4}\right]$ (number density $n_{\text {neo }}=2.7 \mathrm{~nm}^{-3}$ ) in ${ }^{40} \mathrm{Ar}$ at $323 \mathrm{~K}$ and 300 bars (sample II) (O). The curves show the best one-Lorentzian fits $A S_{\text {expt }}(k, \omega)$, with $A$ the absolute normalization factor, and $S_{\text {expt }}(k, \omega)$ given in Eq. (14).

center-of-mass motions are expressed in terms of the intermediate scattering functions $F_{d}(k, t)$ for different molecules, $F_{s}(k, t)$ for a single molecule, and their sum $F(k, t)$, defined by

$$
\begin{gathered}
F_{d}(k, t)=(N-1)\left\langle\exp \{-i \mathbf{k} \cdot \mathbf{R}(0)\} \exp \left\{i \mathbf{k} \cdot \mathbf{R}^{\prime}(t)\right\}\right\rangle, \\
F_{s}(k, t)=\langle\exp \{-i \mathbf{k} \cdot \mathbf{R}(0)\} \exp \{i \mathbf{k} \cdot \mathbf{R}(t)\}\rangle, \\
F(k, t)=F_{d}(k, t)+F_{s}(k, t),
\end{gathered}
$$

where $\mathbf{R}(t)$ and $\mathbf{R}^{\prime}(t)$ denote the center-of-mass positions of two different molecules in the system at time $t$. Then Eq. (3) can be written as

$$
\sigma(k, t)=F_{d}(k, t) u_{\mathrm{coh}}(k)+F_{s}(k, t) v(k, t),
$$

where $u_{\text {coh }}(k)$ is the coherent form factor and $v(k, t)$ the (time dependent) rotational motion contribution of a single molecule. These functions are given by 


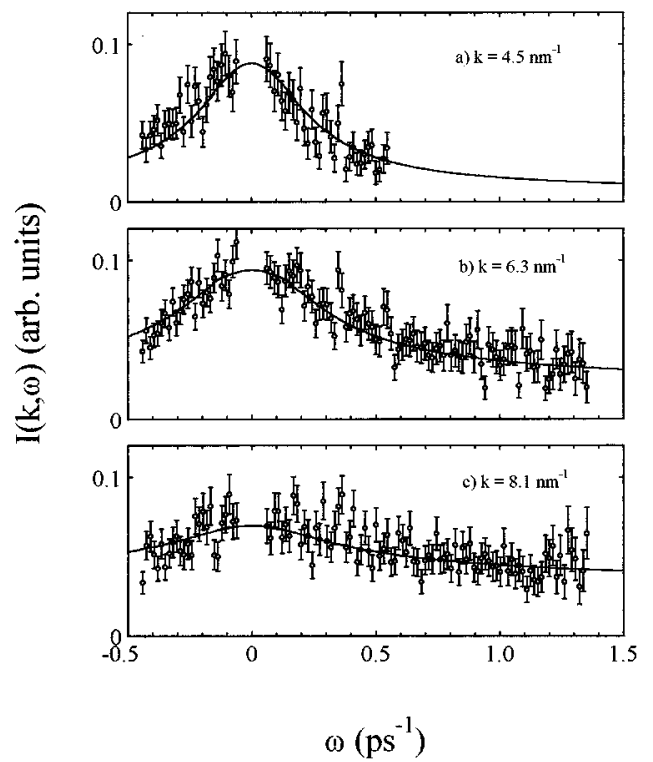

FIG. 3. Neutron intensities $I(k, \omega)$ as functions of frequency $\omega$ at wave numbers $k=4.5$ (a), $6.3(\mathrm{~b})$, and $8.1 \mathrm{~nm}^{-1}$ (c) of neopentane $\left[\mathrm{C}\left(\mathrm{CH}_{3}\right)_{4}\right]$ (number density $n_{\text {neo }}=3 \mathrm{~nm}^{-3}$ ) in ${ }^{40} \mathrm{Ar}$ at $323 \mathrm{~K}$ and 630 bars (sample III) $(\bigcirc)$. The curves show the best twoLorentzian fits $A S_{\text {expt,inc }}(k, \omega)$, with $A$ the absolute normalization factor, and $S_{\text {expt,inc }}(k, \omega)$ given in Eq. (19).

$$
\begin{gathered}
u_{\mathrm{coh}}(k)=\left|\sum_{\nu=1}^{n} b_{\mathrm{coh}}^{\nu} f_{\nu}(k)\right|^{2}, \\
v(k, t)=\sum_{l=0}^{\infty}(2 l+1) a_{l}^{2}(k) F_{l}(t),
\end{gathered}
$$

with

$$
\begin{gathered}
f_{\nu}(k)=\left\langle\exp \left\{i \mathbf{k} \cdot \mathbf{r}_{\nu}\right\}\right\rangle=j_{0}\left(k r_{\nu}\right), \\
a_{l}^{2}(k)=\sum_{\nu, \nu^{\prime}=1}^{n}\left\{b_{\mathrm{coh}}^{\nu} b_{\mathrm{coh}}^{\nu^{\prime}}+b_{\mathrm{inc}}^{\nu^{2}} \delta_{\nu \nu^{\prime}}\right\} j_{l}\left(k r_{\nu}\right) j_{l}\left(k r_{\nu^{\prime}}\right) \\
\times P_{l}\left[\cos \left(\theta_{\nu \nu^{\prime}}\right)\right] .
\end{gathered}
$$

Here $j_{l}(x)$ are spherical Bessel functions and $P_{l}(x)$ Legendre polynomials (of order $l$ ), and $\theta_{\nu \nu^{\prime}}$ is the angle between the positions $\mathbf{r}_{\nu}$ and $\mathbf{r}_{\nu^{\prime}}$ of two nuclei $\nu$ and $\nu^{\prime}$ relative to the center of mass. The rotational motion is characterized by relaxation functions $F_{l}(t)(l=0,1, \ldots)$, which satisfy the conditions $F_{0}(t)=1$ for all $t$, and $F_{l}(0)=1$ for all $l$, and which will be discussed further below. The cross section in Eq. (5) can now be written as an infinite series in $l$, i.e.,

$$
\sigma(k, t)=\sum_{l=0}^{\infty} \sigma_{l}(k, t),
$$

where the subsequent terms $\sigma_{0}(k, t), \sigma_{1}(k, t), \ldots$ arise from the terms labeled $l$ in the expansion of $v(k, t)$ in Eq. (6). The cross section for $s$-wave scattering $(l=0)$, dominant at small $k$, is given by

$$
\sigma_{0}(k, t)=F(k, t) u_{\text {coh }}(k)+F_{s}(k, t) u_{\text {inc }}(k),
$$

TABLE II. Bound scattering lengths of atoms present in the samples [13].

\begin{tabular}{ccc}
\hline \hline Atom & $\begin{array}{l}b_{\text {coh }} \\
(\mathrm{fm})\end{array}$ & $\begin{array}{l}b_{\text {inc }} \\
(\mathrm{fm})\end{array}$ \\
\hline $\mathrm{H}$ & -3.74 & 25.27 \\
$\mathrm{D}$ & 6.67 & 4.04 \\
$\mathrm{C}$ & 6.65 & 0 \\
${ }^{40} \mathrm{Ar}$ & 1.83 & 0 \\
\hline \hline
\end{tabular}

with the incoherent form factor of one molecule,

$$
u_{\text {inc }}(k)=\sum_{\nu=1}^{n} b_{\text {inc }}^{\nu^{2}} j_{0}^{2}\left(k r_{\nu}\right) \text {. }
$$

The higher order terms $(l \geqslant 1)$ in Eq. (8) are given by

$$
\sigma_{l}(k, t)=(2 l+1) a_{l}^{2}(k) F_{s}(k, t) F_{l}(t) .
$$

Thus the $s$-wave scattering contribution in Eq. (9) involves the coherent and incoherent form factors $u_{\text {coh }}(k)$ and $u_{\text {inc }}(k)$ of a single molecule and the translational motions of all molecules [the collective, coherent $F(k, t)]$ and that of a single molecule [the single particle, incoherent $F_{s}(k, t)$ ]. Higher order terms in the partial wave expansion $(l \geqslant 1)$ include the products of translational and rotational scattering functions $F_{s}(k, t) F_{l}(t)$, with intensities proportional to $(2 l+1) a_{l}^{2}(k)$ [cf. Eq. (11)]. For fixed $k$, this is a rapidly decreasing series in $l$, so that only the first few terms in Eq. (8) need to be retained.

In the next section we show that in the range of $k$ values involved in the present experiments $\left(k<12 \mathrm{~nm}^{-1}\right)$ for $\mathrm{C}\left(\mathrm{CD}_{3}\right)_{4}$ only $\sigma_{0}(k, t)$ contributes and that $u_{\text {coh }}(k) \gg u_{\text {inc }}(k)$. Thus for the deuterated samples I and II one observes in practice the collective translational motion which is described by $F(k, t)$ [cf. Eq. (9)]. For $\mathrm{C}\left(\mathrm{CH}_{3}\right)_{4}$ (sample III) the second term in Eq. (9) $(l=0)$ and the term for $l=1$ in Eq. (8) are needed, i.e., the translational motion of single neopentane molecules alone $\left[F_{s}(k, t)\right]$ and the combination of translational and rotational motions of single neopentane molecules $\left[F_{s}(k, t) F_{1}(t)\right]$.

\section{RESULTS}

\section{A. Samples I and II}

We start from Eqs. (8), (9), and (11). The form factors $u_{\text {coh }}(k)$ and $u_{\text {inc }}(k)$ of neopentane can be calculated using the bound scattering lengths $b_{\text {coh }}^{\mathrm{C}}, b_{\text {inc }}^{\mathrm{C}}, b_{\text {coh }}^{\mathrm{D}}$, and $b_{\text {inc }}^{\mathrm{D}}$ (cf. Table II) [13], and distances $r_{C}=0.154 \mathrm{~nm}$ and $r_{D}=r_{H}=0.216 \mathrm{~nm}$ [14]. We plot the form factors $u_{\text {coh }}(k)$ and $u_{\text {inc }}(k)$ in Fig. 4. One observes that $u_{\text {coh }}(k) \gg u_{\text {inc }}(k)$ for all $k$. The influence of the intermolecular structure factor $S(k)[=F(k, 0)]$ can be estimated with the Percus-Yevick theory for binary hard sphere fluids. We use effective hard sphere diameters $\sigma_{\mathrm{HS}, \mathrm{Ar}}=0.335 \mathrm{~nm}$ for $\operatorname{Ar}[4]$ and estimate $\sigma_{\mathrm{HS}, \mathrm{nec}}=0.57 \pm 0.02$ $\mathrm{nm}$ for neopentane from LJ potentials $[8,9]$. The densities of the components are given in Table I. The structure factors $S_{\mathrm{HS}}^{i}(k)$ calculated for sample $i=\mathrm{I}$ and II are plotted in Fig. 5. The product $S_{\mathrm{HS}}^{i}(k) u_{\mathrm{coh}}(k)$ is shown for both samples in Fig. 4. For the terms with $l \geqslant 1$ in Eq. (11) the intensities are 


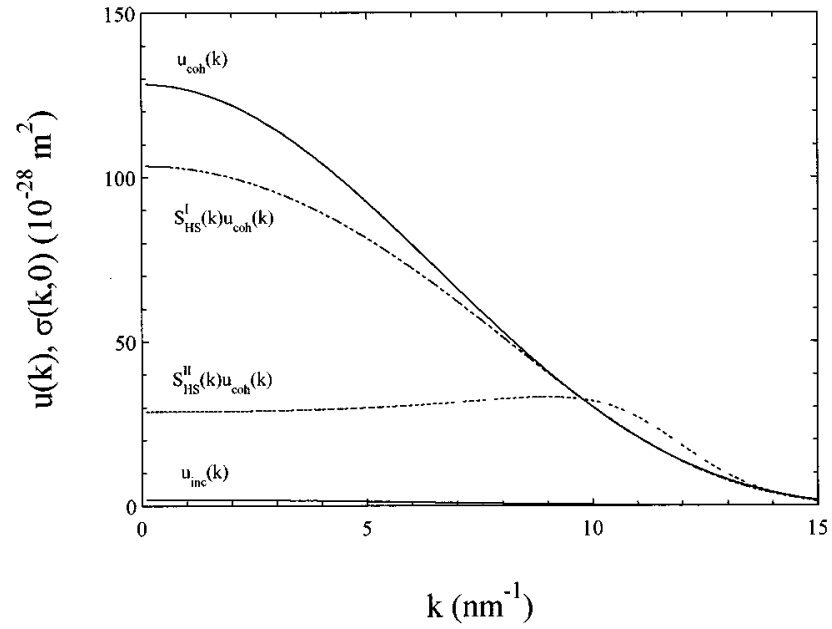

FIG. 4. The coherent and incoherent form factors $u_{\text {coh }}(k)$ [cf. Eq. (6)] and $u_{\text {inc }}(k)$ [cf. Eq. (10)] of neopentane $\left[\mathrm{C}\left(\mathrm{CD}_{3}\right)_{4}\right]$ as functions of wave number $k$. The dashed lines show the theoretical intensities $\sigma(k, 0)=S_{\mathrm{HS}}^{i}(k) u_{\mathrm{coh}}(k)$ calculated for equivalent hard sphere fluids for sample $i=\mathrm{I}$ and II.

given by Eq. (7). The first two $(l=1,2)$ coherent terms cancel due to the tetrahedral symmetry (destructive interference). The incoherent terms for $l \geqslant 1$ are of the same order as $u_{\text {inc }}(k)$, and can thus be neglected in this $k$ range. Therefore we retain only the $l=0$ contribution from Eqs. (8), (9), and (11), so that for samples I and II,

$$
\sigma(k, t)=\sigma_{0}(k, t)=F(k, t) u_{\mathrm{coh}}(k)
$$

( samples I,II).

The time dependence of $F(k, t)$ is given by [with $S(k)$ $=F(k, 0)]$

$$
F(k, t)=S(k) \exp \left[-\omega_{H}(k) t\right]
$$

with $\omega_{H}(k)$ the decay rate of density fluctuations with wave number $k$ due to collective motion of the neopentane molecules in Ar. Fourier transformation [cf. Eq. (2)] of Eq. (12) with $F(k, t)$ given by Eq. (13) yields the experimental coherent dynamic structure factor

$$
S_{\text {expt }}(k, \omega)=\frac{S_{\text {expt }}(k)}{\pi} \frac{\omega_{H}(k)}{\omega_{H}(k)^{2}+\omega^{2}},
$$

with

$$
S_{\text {expt }}(k)=S(k) u_{\text {coh }}(k) \text {, }
$$

which is a Lorentzian line in $\omega$ with half-width $\omega_{H}(k)$ and intensity $S_{\text {expt }}(k)$. The experimental data $I(k, \omega)$ were fitted with one Lorentzian with free intensity $I(k)=A S_{\text {expt }}(k)$, and half-width $\omega_{H}(k)$. The fits are plotted (full lines) in Fig. 1 for sample I and Fig. 2 for sample II. The results for $S_{\text {expt }}(k)$ for samples I and II are shown in Fig. 6. The intensities $S_{\text {expt }}(k)$ as functions of $k$ are in reasonable agreement with the theoretical functions $S_{\mathrm{HS}}(k) u_{\mathrm{coh}}(k)$ shown in Fig. 4. We plot the static structure factor $S(k)=S_{\text {expt }}(k) / u_{\text {coh }}(k)$ for sample II in Fig. 7. $S(k)$ is in good agreement with $S_{\mathrm{HS}}(k)$ (solid line, cf. Fig. 5) for $k>6 \mathrm{~nm}^{-1}$. Differences between the experimental

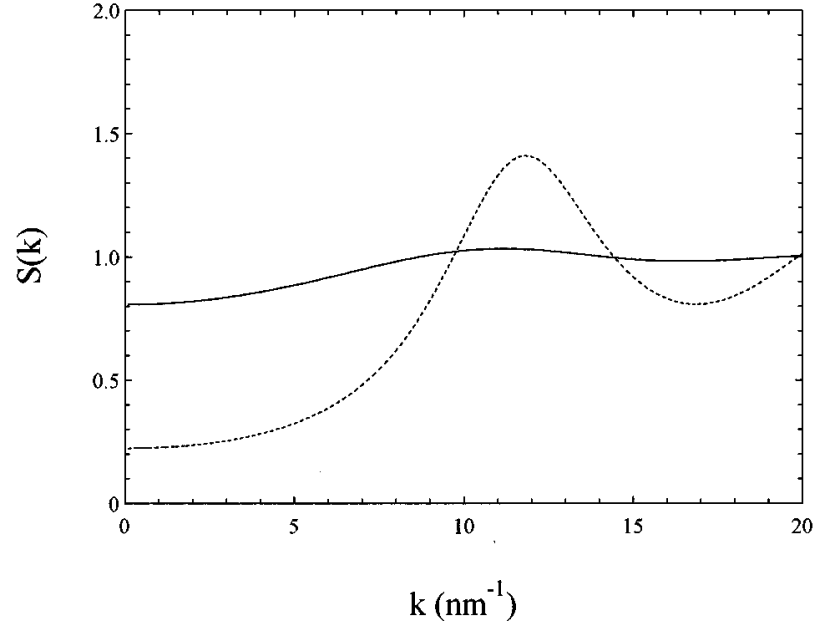

FIG. 5. Equivalent hard sphere static structure factors $S_{\mathrm{HS}}(k)$ for neopentane in Ar as functions of wave number $k$. Solid line: sample I $\left(n_{\text {neo }}=0.4 \mathrm{~nm}^{-3}\right)$; dashed line: sample II $\left(n_{\text {neo }}=2.7 \mathrm{~nm}^{-3}\right)$.

intensities and those calculated for hard spheres in Figs. 6 and 7 will be discussed in Secs. V A and V B. The absolute normalization factor $A$ is determined by overlap of $S_{\text {expt }}(k)$ with these theoretical functions $S_{\mathrm{HS}}(k) u_{\mathrm{coh}}(k)$.

The half-widths $\omega_{H}(k)$ are shown in Fig. 8. For $k<5$ $\mathrm{nm}^{-1}$ the half-widths are described by $\omega_{H}(k)=D_{c} k^{2}$, with $D_{c}=(21 \pm 1) \times 10^{-9} \mathrm{~m}^{2} \mathrm{~s}^{-1}$, and $(13 \pm 2) \times 10^{-9} \mathrm{~m}^{2} \mathrm{~s}^{-1}$ the collective diffusion coefficients for neopentane in these samples, respectively. For $k>8 \mathrm{~nm}^{-1}$ the half-width of the narrowest line of the low-concentration sample (sample I) shows a crossover to a linear behavior of $\omega_{H}(k)$ in $k$. The widths of the Lorentzians obtained from the high-
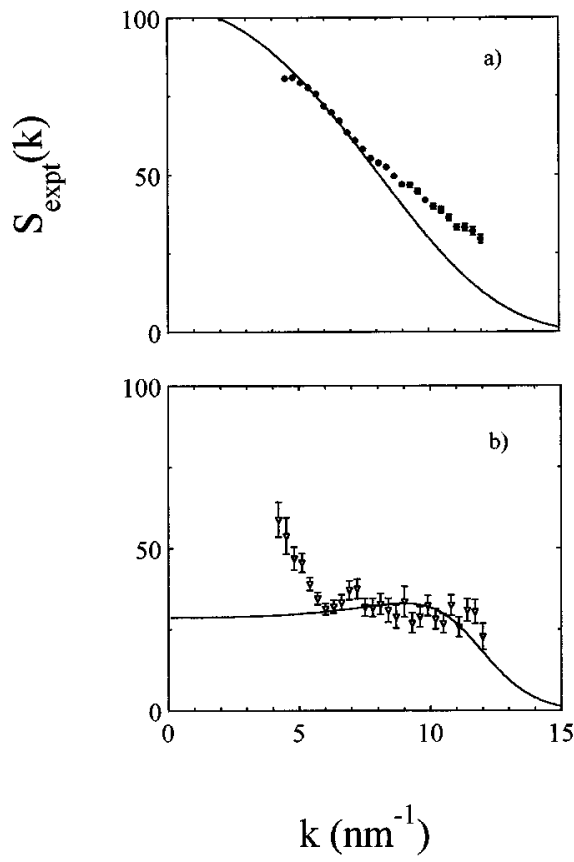

FIG. 6. Experimental intensities $S_{\text {expt }}(k)$ [cf. Eq. (15)] as functions of wave number $k$ for sample I [(a), $]$ and sample II $[(\mathrm{b}), \nabla]$. The solid lines represent the theoretical intensities for equivalent hard sphere mixtures. Differences between experiment and theory are discussed in the text. 


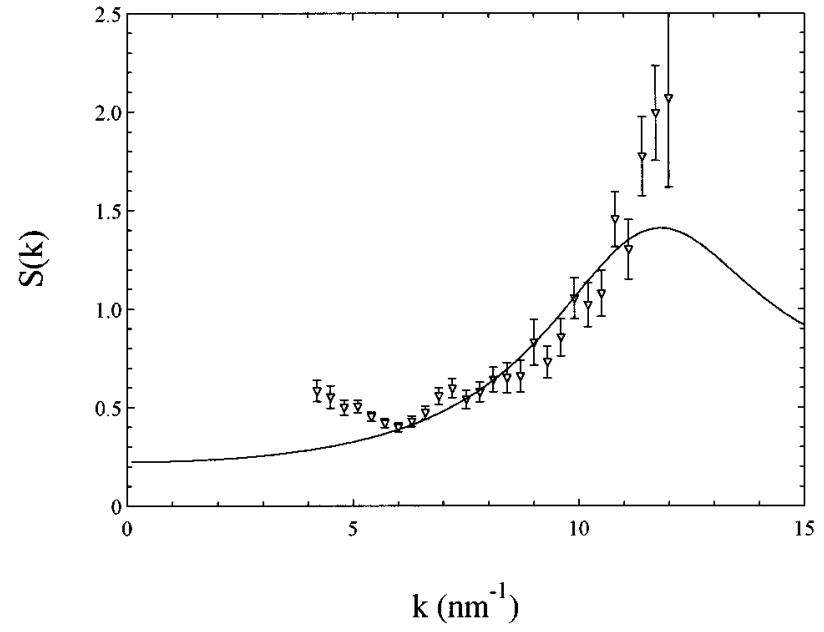

FIG. 7. Static structure factor $S(k)=S_{\text {expt }}(k) / u_{\text {coh }}(k)$ (cf. Figs. 4 and 6) for sample II $(\nabla)$ as a function of wave number $k$. The line is theoretical for equivalent hard spheres (cf. dashed line in Fig. 5). Differences between experiment and theory are discussed in the text.

concentration-sample (sample II) data, however, show a plateau for $8<k<12 \mathrm{~nm}^{-1}$ at height $\omega_{H}(k) \approx 0.7 \mathrm{ps}^{-1}$. For $k<10 \mathrm{~nm}^{-1}$, the half-widths $\omega_{H}(k)$ from experiment are in good agreement with the half-widths calculated with the RET (solid lines in Fig. 8). Differences between the observed half-widths and those from the RET, that appear mainly at higher wave numbers $k>10 \mathrm{~nm}^{-1}$, will be analyzed in the discussion.


FIG. 8. Half-widths $\omega_{H}(k)$ of $S_{\text {expt }}(k, \omega)$ [(a), cf. Eq. (14)] and $\omega_{H, s}(k)$ of $S_{\text {expt,inc }}(k, \omega)$ [(b), cf. Eq. (19)] as functions of wave number $k$ for neopentane in ${ }^{40} \mathrm{Ar}$. (a) $\mathrm{C}\left(\mathrm{CD}_{3}\right)_{4}$ : experiment for $n_{\text {neo }}=0.4 \mathrm{~nm}^{-3}(\boldsymbol{O})$, for $n_{\text {neo }}=2.7 \mathrm{~nm}^{-3}(\nabla)$. The solid curves are from the Enskog theory. The dashed line shows the ideal gas behavior [cf. Eq. (21)]. (b) $\mathrm{C}\left(\mathrm{CH}_{3}\right)_{4}$ : experiment for $n_{\text {neo }}=3 \mathrm{~nm}^{-3}$ (ם). The solid line is the best fit $\omega_{H, s}(k)=D_{s} k^{2}$ with self-diffusion coefficient $D_{s}=(9 \pm 2) \times 10^{-9} \mathrm{~m}^{2} \mathrm{~s}^{-1}$.

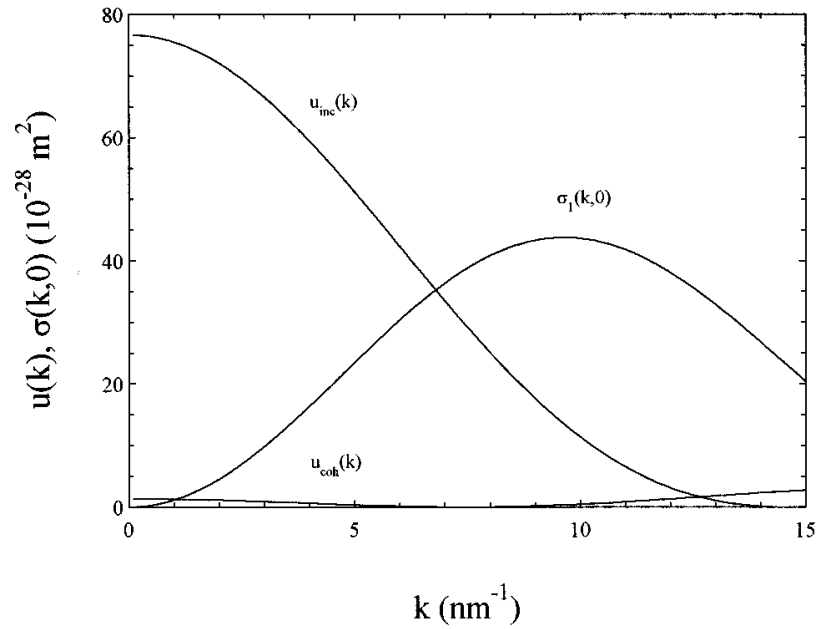

FIG. 9. The coherent and incoherent form factors $u_{\text {coh }}(k)$ [cf.

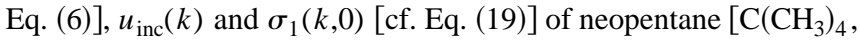
sample III] as functions of wave number $k$.

\section{B. Sample III}

For $\mathrm{C}\left(\mathrm{CH}_{3}\right)_{4}$ the scattering is almost completely due to the incoherent scattering from the protons since $b_{\mathrm{coh}}^{\mathrm{H}}=-3.74 \mathrm{fm}$ and $b_{\mathrm{inc}}^{\mathrm{H}}=25.27 \mathrm{fm}$ (cf. Table II). It follows from Eqs. (8), (9), and (11) that the total scattering cross section is given by

$$
\begin{aligned}
\sigma(k, t)= & F(k, t) u_{\mathrm{coh}}(k)+F_{s}(k, t) u_{\mathrm{inc}}(k) \\
& +36\left(b_{\mathrm{inc}}^{\mathrm{H}}\right)^{2} j_{1}\left(k r_{H}\right)^{2} F_{s}(k, t) F_{1}(t)+\cdots
\end{aligned}
$$

( sample III).

In Fig. 9 we plot the form factors $u_{\text {coh }}(k)$ and $u_{\text {inc }}(k)$ and the cross section $\sigma_{1}(k, 0)$. The coherent term $F(k, t) u_{\text {coh }}(k)$ contributes less than $5 \%$ to the total scattering cross section due to destructive interference of the positive and negative coherent scattering lengths of $\mathrm{C}$ and $\mathrm{H}$, respectively, and thus can be neglected. Hence we can describe $\sigma(k, t)$ by

$$
\sigma(k, t)=F_{s}(k, t)\left\{u_{\text {inc }}(k)+36\left(b_{\text {inc }}^{\mathrm{H}}\right)^{2} j_{1}\left(k r_{H}\right)^{2} F_{1}(t)+\cdots\right\}
$$

( sample III),

where the higher terms $l=2, \ldots$ may be neglected.

For the rotational motion we assume rotational diffusion, since the collision frequency is high compared to the angular frequency of a neopentane molecule in thermal equilibrium. Therefore $F_{s}(k, t)$ and $F_{l}(t)$ are given by

$$
\begin{aligned}
& F_{l}(t)=\exp \left[-l(l+1) D_{r} t\right], \\
& F_{s}(k, t)=\exp \left[-\omega_{H, s}(k) t\right],
\end{aligned}
$$

with $D_{r}$ the rotational diffusion coefficient, and $\omega_{H, s}(k)$ the decay rate of density fluctuations due to the translational self-motion. Fourier transformation [cf. Eq. (2)] of Eq. (17) with $F_{s}(k, t)$ and $F_{l}(t)$ given by Eq. (18) leads to a sum of Lorentzians, with intensities given by $(2 l+1) j_{l}\left(k r_{H}\right)^{2}$ (see Fig. 9) and half-widths $\omega_{H, l}(k)=\omega_{H, s}(k)+l(l+1) D_{r}$. It appears that only the first two terms for $l=0,1$ were narrow 
TABLE III. Viscosity $\eta$ of Ar at $T=323 \mathrm{~K}$ and sample pressure $p$, Stokes-Einstein $D_{\mathrm{SE}}$, Enskog $D_{E}$, and experimental $D_{\text {expt }}$ diffusion coefficients for samples I, II, and III.

\begin{tabular}{|c|c|c|c|c|c|}
\hline Sample No. & $\begin{array}{c}p \\
\left(10^{5} \mathrm{~Pa}\right)\end{array}$ & $\begin{array}{c}\eta \\
\left(10^{-5} \mathrm{~Pa} \mathrm{~s}\right)\end{array}$ & $\underset{\left(10^{-9} \mathrm{~m}^{2} \mathrm{~s}^{-1}\right)}{D_{\mathrm{SE}}}$ & $\begin{array}{c}D_{E} \\
\left(10^{-9} \mathrm{~m}^{2} \mathrm{~s}^{-1}\right)\end{array}$ & $\begin{array}{c}D_{\text {expt }} \\
\left(10^{-9} \mathrm{~m}^{2} \mathrm{~s}^{-1}\right)\end{array}$ \\
\hline I & 300 & 3.56 & 23 & 22 & $21 \pm 1$ \\
\hline II & 300 & 3.56 & 23 & 14 & $13 \pm 2$ \\
\hline III & 630 & 5.09 & 16 & 7 & $9 \pm 2$ \\
\hline
\end{tabular}

(and thus intense) enough to be observed in the experimental $\omega$ window $-0.5<\omega<1.4 \mathrm{ps}^{-1}$. Hence

$$
\begin{aligned}
S_{\text {expt,inc }}(k, \omega)= & \frac{12\left(b_{\mathrm{inc}}^{\mathrm{H}}\right)^{2}}{\pi}\left\{j_{0}\left(k r_{H}\right)^{2} \frac{\omega_{H, s}(k)}{\omega_{H, s}(k)^{2}+\omega^{2}}\right. \\
& \left.+3 j_{1}\left(k r_{H}\right)^{2} \frac{\omega_{H, s}(k)+2 D_{r}}{\left[\omega_{H, s}(k)+2 D_{r}\right]^{2}+\omega^{2}}\right\} .
\end{aligned}
$$

The data $I(k, \omega)$ were fitted with $I(k, \omega)=A S_{\text {expt,inc }}(k, \omega)$ with free $\omega_{H, s}(k), D_{r}$, and $A$ the absolute normalization factor. The fits are shown in Fig. 3 (full lines). The data are best fitted with $D_{r}=0.8 \pm 0.3 \mathrm{ps}^{-1}$. Due to the limited experimental frequency window, this result is not very accurate. The results for $\omega_{H, s}(k)$ are shown in Fig. 8. The half-widths are fitted with $\omega_{H, s}(k)=D_{s} k^{2}$ which gives a self-diffusion coefficient $D_{s}=(9 \pm 2) \times 10^{-9} \mathrm{~m}^{2} \mathrm{~s}^{-1}$.

\section{DISCUSSION}

By inelastic neutron scattering we obtain the experimental dynamic structure factors $S_{\text {expt }}(k, \omega)$ for three dense binary mixtures of neopentane in ${ }^{40} \mathrm{Ar}$ at $T=323 \mathrm{~K}$ which we denote as samples I, II, and III for increasing neopentane number densities $n_{\text {neo }}$ (cf. Table I). Samples I and II consist of deuterated neopentane $\left[\mathrm{C}\left(\mathrm{CD}_{3}\right)_{4}\right]$ and sample III of hydrogenated neopentane $\left[\mathrm{C}\left(\mathrm{CH}_{3}\right)_{4}\right]$. The coherent scattering lengths $b_{\mathrm{coh}}^{\mathrm{C}}$ and $b_{\mathrm{coh}}^{\mathrm{D}}$ for $\mathrm{C}$ and $\mathrm{D}$ are both positive, while $b_{\mathrm{coh}}^{\mathrm{H}}$ for $\mathrm{H}$ is negative (cf. Table II), resulting in constructive interference for samples I and II and destructive interference for sample III for coherent scattering. Furthermore for D, the incoherent scattering length $b_{\mathrm{inc}}^{\mathrm{D}}<b_{\text {coh }}^{\mathrm{D}}$, whereas for $\mathrm{H}$ $b_{\text {inc }}^{\mathrm{H}} \gg b_{\text {coh }}^{\mathrm{H}}$ (cf. Table II). Therefore we observe for samples I and II mainly the coherent motions of the neopentane molecules, while for sample III, the translational and rotational motions of a single neopentane molecule are observed. For all three samples the contribution of the ${ }^{40} \mathrm{Ar}$ atoms to the spectra $S_{\text {expt }}(k, \omega)$ can be neglected, since the ${ }^{40} \mathrm{Ar}$ cross section $\left(b_{\mathrm{coh}}^{\mathrm{Ar}}\right)^{2}=0.033 \times 10^{-28} \mathrm{~m}^{2}$ (cf. Table II), which is orders of magnitude smaller than the values $\sigma(k, 0)>10 \times 10^{-28} \mathrm{~m}^{2}$ for all neopentane samples (cf. Figs. 4 and 9).

The experimental dynamic structure factors $S_{\text {expt }}(k, \omega)$ for $4<k<12 \mathrm{~nm}^{-1}$ for samples I and II consist of one Lorentzian in $\omega$, with half-width at half maximum $\omega_{H}(k)$ and intensity $S_{\text {expt }}(k)$ [cf. Eq. (14)]. For $4<k<8 \mathrm{~nm}^{-1}$ the experimental dynamic structure factor $S_{\text {expt,inc }}(k, \omega)$ for sample III consists of two Lorentzians, the narrower one due to the translational motion of single neopentane molecules alone, and the second (broader) one due to a combination of trans- lational and rotational motions of single neopentane molecules [cf. Eq. (19)]. We discuss the results for the different samples separately.

\section{A. Sample I \\ 1. Dynamics}

For the half-widths $\omega_{H}(k)$ of the dynamic structure factors $S_{\text {expt }}(k, \omega)$ of sample I (cf. Sec. IV A) we find for $k<5$ $\mathrm{nm}^{-1}$ that $\omega_{H}(k)=D_{c} k^{2}$ with $D_{c}=(21 \pm 1) \times 10^{-9} \mathrm{~m}^{2} \mathrm{~s}^{-1}$ the collective diffusion coefficient of neopentane in $\mathrm{Ar}$ (cf. Fig. 8). Since reduced density $n^{*}=n_{\text {neo }} \sigma_{\text {neo }}^{3}=0.07$ of neopentane is low (cf. Table I), we may compare the diffusion coefficient $D_{c}$ with the continuum Stokes-Einstein description for the diffusion coefficient of one neopentane molecule (the solute) at infinite dilution,

$$
D_{\mathrm{SE}}=\frac{k_{B} T}{3 \pi \eta \sigma_{\mathrm{neo}}},
$$

with $\eta$ the shear viscosity of Ar (the solvent). At 300 bars and $323 \mathrm{~K}, \quad \eta=3.56 \times 10^{-5} \quad \mathrm{~Pa} \mathrm{~s}$ [14], yielding $D_{\mathrm{SE}}=23 \times 10^{-9} \mathrm{~m}^{2} \mathrm{~s}^{-1}$. We conclude that the StokesEinstein description is in reasonable agreement with experiment.

For $k \leqq 8 \mathrm{~nm}^{-1}$ the half-widths $\omega_{H}(k)$ for sample I are also in good agreement with those calculated with the RET for an equivalent binary hard sphere mixture, yielding $D_{E}=22 \times 10^{-9} \mathrm{~m}^{2} \mathrm{~s}^{-1}$ (cf. Table III). At higher wave numbers $k \gtrsim 8 \mathrm{~nm}^{-1}$ the half-widths $\omega_{H}(k)$ are slightly higher than those from RET, and approach the ideal gas behavior

$$
\omega_{H}^{\mathrm{id}}(k)=\left(\frac{2 \ln 2 k_{B} T}{m_{\text {neo }}}\right)^{0.5} k,
$$

with $m_{\text {neo }}=1.40 \times 10^{-25} \mathrm{~kg}$ the mass of one neopentane $\left[\mathrm{C}\left(\mathrm{CD}_{3}\right)_{4}\right]$ molecule (dashed line in Fig. 8). Equation (21) is valid for $k \rightarrow \infty$ for particles interacting through soft potentials, while the RET is exact for $k \rightarrow \infty$ and hard spheres. Therefore the difference seen in Fig. 8 between the RET and experiment implies that neopentane is not a perfect hard sphere [16]. We still conclude that the RET is relevant for low concentrations at diameter ratios $\sigma_{1} / \sigma_{2} \leqslant 1.7$.

\section{Structure}

The intensity $S_{\text {expt }}(k)$ obtained from the Lorentzian fits to the data of sample I are only in qualitative agreement with the theoretical intensity $S_{\mathrm{HS}}(k) u_{\mathrm{coh}}(k)$ (cf. Fig. 6). This is caused by the assumption that $S_{\text {expt }}(k, \omega)$ can be described with a Lorentzian for all $\omega$, and not only in the (limited) experimental $\omega$ window $-0.7<\omega<2.0 \mathrm{ps}^{-1}$. For large wave 




FIG. 10. The equivalent hard sphere dynamic structure factor $S_{\mathrm{HS}}(k, \omega)$ from RET for sample I (solid line), at wave number $k=12 \mathrm{~nm}^{-1}$, as a function of frequency $\omega$. Lorentzian (upper dashed line) and Gaussian (lower dashed line) fits to $S_{\mathrm{HS}}(k, \omega)$ for $\omega<2.0 \mathrm{ps}^{-1}$ (vertical line), the experimental frequency window. The fitted half-widths are accurate, but the fitted intensities deviate systematically.

numbers $k$ and large frequencies $\omega, S_{\text {expt }}(k, \omega)$ is described by a Gaussian function in $\omega$ with half-width $\omega_{H}^{\text {id }}(k)$ given by Eq. (21) [2]. To estimate the systematic error we make by fitting $S_{\text {expt }}(k, \omega)$ with a Lorentzian in $\omega$ at large wave numbers $k$ and $|\omega|<2.0 \mathrm{ps}^{-1}$ we use the equivalent hard sphere dynamic structure factor $S_{\mathrm{HS}}(k, \omega)$ at $k=12 \mathrm{~nm}^{-1}$, calculated from RET, with intensity $S_{\mathrm{HS}}\left(k=12 \mathrm{~nm}^{-1}\right)=1$ (cf. solid line in Fig. 5) and $\omega_{H}\left(k=12 \mathrm{~nm}^{-1}\right)=1.95 \mathrm{ps}^{-1}$ (cf. Fig. 8). We fit $S_{\mathrm{HS}}(k, \omega)$ for $|\omega|<2.0 \mathrm{ps}^{-1}$ with a Lorentzian with free intensity $L$ and half-width $\omega_{H}$ (see Fig. 10). We find $L=1.5$ and $\omega_{H}=2.0 \mathrm{ps}^{-1}$. Thus we overestimate the intensity $S_{\mathrm{HS}}(k)$ by a factor 1.5 , while the estimation of the half-width $\omega_{H}$ is rather accurate. Also, for a Gaussian $G(\omega)$ (area $G$, half-width $\omega_{H}^{G}$ ) and Lorentzian $L(\omega)$ (area $L$, half-width $\left.\omega_{H}^{L}\right)$ with equal maxima $[G(0)=L(0)]$ and equal halfwidths $\omega_{H}^{G}=\omega_{H}^{L}$, the ratio of the areas $L / G=(\pi \ln 2)^{0.5} \approx 1.5$. This is exactly what we find for the largest wave number $k=12 \mathrm{~nm}^{-1}$ in the present experiments. The overestimation of the fitted intensity $S_{\text {expt }}(k)$ decreases with decreasing $k$, since the spectra become narrower and the Lorentzian approximation becomes increasingly better for all $\omega$ (cf. Fig. $1)$. Thus the deviations of the experimental intensities $S_{\text {expt }}(k)$ from the theoretical intensity $S_{\mathrm{HS}}(k) u_{\text {coh }}(k)$ in Fig. 6 are due to the finite experimental $\omega$ window.

\section{B. Sample II}

\section{Dynamics}

The experimental half-widths $\omega_{H}(k)$ of sample II agree reasonably well with those calculated from the RET (cf. Fig. 8). For $k<8 \mathrm{~nm}^{-1}, \omega_{H}(k)=D_{c} k^{2}$ with $D_{c}=(13 \pm 2) \times 10^{-9}$ $\mathrm{m}^{2} \mathrm{~s}^{-1}$ the collective diffusion coefficient of neopentane, which agrees with the Enskog value $D_{E}=14 \times 10^{-9} \mathrm{~m}^{2} \mathrm{~s}^{-1}$ (cf. Table III). We note in Table III that for sample II, $D_{c}$ is very different from $D_{\mathrm{SE}}$ due to the fact that the reduced density of neopentane is very high (cf. Table I). For $k>8$

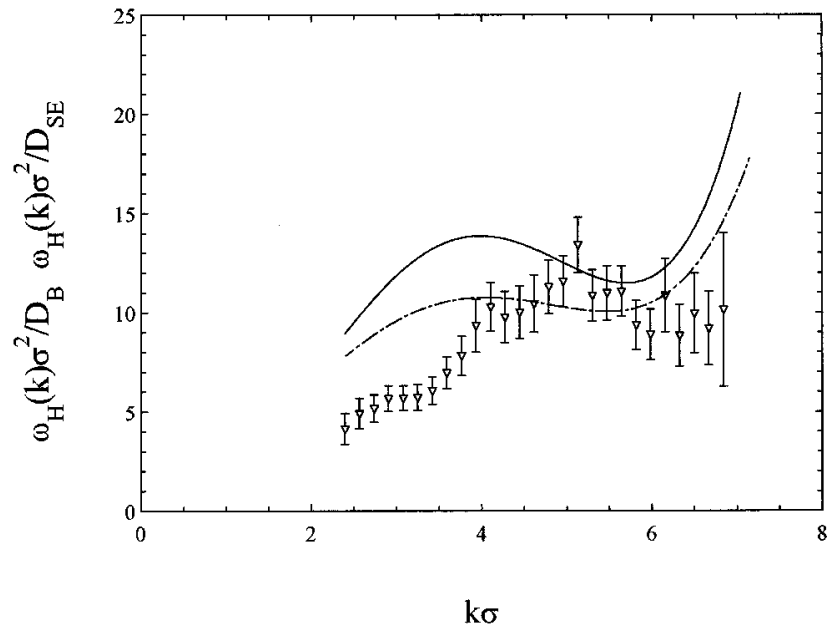

FIG. 11. Reduced half-widths $\omega_{H}(k) \sigma^{2} / D_{B}$ of pure ${ }^{36} \mathrm{Ar}$ $\left(n^{*}=0.51, \sigma=0.335 \mathrm{~nm}, \sigma^{2} / D_{B}=3.6 \mathrm{ps}\right.$ [17]; dashed line) and $\omega_{H}(k) \sigma^{2} / D_{\mathrm{SE}}$ of $\mathrm{C}\left(\mathrm{CD}_{3}\right)_{4}$ in ${ }^{40} \mathrm{Ar}\left[n_{\text {neo }}^{*}=0.5, \sigma=0.57 \mathrm{~nm}\right.$, $\sigma^{2} / D_{\mathrm{SE}}=14$ ps (sample II); $\left.\nabla\right]$ and of latex dispersed in benzene $\left(n^{*}=0.64, \sigma=219 \mathrm{~nm}, \sigma^{2} / D_{\mathrm{SE}}=16 \mathrm{~ms}[18]\right.$; solid line) as a function of reduced wave number $k \sigma$.

$\mathrm{nm}^{-1}$, the theoretical and experimental half-widths show a behavior reminiscent of "de Gennes narrowing" in simple fluids and dense colloidal suspensions. For dense simple fluids and concentrated colloidal suspensions one observes a flattening or a minimum in the coherent half-width $\omega_{H}(k)$ for $k$ values near $k^{*}=2 \pi / \sigma$ where the static structure factor $S(k)$ has its first maximum. We compare the dynamics of neopentane in Ar at $n_{\text {neo }}^{*}=n_{\text {neo }} \sigma_{\text {neo }}^{3}=0.5$ (cf. Table I) with that of simple fluids and dense colloidal suspensions at similar reduced densities $n^{*}=n \sigma^{3}$. As an example for simple fluids, we use the half-widths $\omega_{H}(k)$ of $S(k, \omega)$ for pure ${ }^{36} \mathrm{Ar}$ at $n^{*}=0.51\left(T=212 \mathrm{~K}, n=13.5 \mathrm{~nm}^{-3}\right.$, and effective hard sphere diameter $\sigma=0.335 \mathrm{~nm}$ [17]. For colloids, we take the data of Fijnaut et al. for a concentrated latex dispersion $[\sigma=219 \mathrm{~nm}$, determined from $S(k)]$ with reduced density $n^{*}=0.64$ [18]. To compare the half-widths quantitatively, we plot the reduced half-widths $\omega_{H}(k) \sigma^{2} / D$ as a function of $k \sigma$ in Fig. 11 for neopentane, Ar, and latex. $\sigma^{2} / D$ is the time in which one particle diffuses (with diffusion coefficient $D$ ) over a distance equal to its diameter $\sigma$. For simple fluids we use $D=D_{B}$ the Boltzmann diffusion coefficient of a single particle. For the $\mathrm{Ar}$ data here, $D_{B}=3.1 \times 10^{-8} \mathrm{~m}^{2} \mathrm{~s}^{-1}$, and $\sigma^{2} / D_{B}=3.6 \mathrm{ps}$. For neopentane and colloidal suspensions we use $D=D_{\mathrm{SE}}$, the StokesEinstein diffusion coefficient given by Eq. (20). For the latex dispersions, $D_{\mathrm{SE}}=3.0 \times 10^{-12} \mathrm{~m}^{2} \mathrm{~s}^{-1}$ is measured, yielding $\sigma^{2} / D_{\mathrm{SE}}=16 \mathrm{~ms}$. For neopentane we find $D_{\mathrm{SE}}=23 \times 10^{-9}$ $\mathrm{m}^{2} \mathrm{~s}^{-1}$ (cf. Table III), so that $\sigma^{2} / D_{\mathrm{SE}}=14$ ps. The quantitative agreement between the reduced half-widths $\omega_{H}(k) \sigma^{2} / D$ as a function of $k \sigma$ for ${ }^{36} \mathrm{Ar}$, neopentane, and latex in Fig. 11 is reasonable for $k \sigma \approx 6$, i.e., around the main peak in the static structure factor $S(k)$. That means that for these length scales the dynamics of the solute particles is largely determined by the structure of the solute particles themselves. Especially for binary mixtures, this is a remarkable result, since the diameter ratio $\sigma_{1} / \sigma_{2}$ of solute and solvent particles is not very large ( 1.7 for the present samples) and the time 
scale $\sigma^{2} / D$ (14 ps for sample II) is much nearer to that of simple fluids ( $3.6 \mathrm{ps}$ for ${ }^{36} \mathrm{Ar}$ ) than to that for colloids (ms for the latex suspension). The influence of the finite diameter ratio $\sigma_{1} / \sigma_{2}$ is observable at smaller wave numbers $k \sigma<4$ where the half-widths for neopentane in Ar are smaller than those for pure Ar and colloids (cf. Fig. 11).

\section{Structure}

The experimentally determined static structure factor $S(k)=S_{\text {expt }}(k) / u_{\text {coh }}(k)$ is in good agreement with the static structure factor for hard spheres $S_{\mathrm{HS}}(k)$ for $k>6 \mathrm{~nm}^{-1}$, but larger for $k<6 \mathrm{~nm}^{-1}$ (cf. Fig. 7). Similar effects in $S(k)$ have been observed in He-Ar mixtures at $T=160 \mathrm{~K}[4,6]$, i.e., just above the critical temperature $T_{c}=151 \mathrm{~K}$ above which the mixture is fully miscible. Thus we attribute the increase of $S(k)$ for $k \rightarrow 0$ to attractive forces between the neopentane molecules, which are absent for hard spheres.

\section{Sample III}

\section{Translational dynamics}

For the half-widths $\omega_{H}(k)$ of the narrower Lorentzian line we find $\omega_{H}(k)=D_{s} k^{2}$ with translational self-diffusion coefficient $D_{s}=(9 \pm 2) \times 10^{-9} \mathrm{~m}^{2} \mathrm{~s}^{-1}$. This is in reasonably good agreement with the Enskog theory, which yields $D_{E}=7 \times 10^{-9} \mathrm{~m}^{2} \mathrm{~s}^{-1}$. We now compare $D_{s}=9 \times 10^{-9}$ $\mathrm{m}^{2} \mathrm{~s}^{-1}$ with the Stokes-Einstein diffusion coefficient $D_{\mathrm{SE}}=16 \times 10^{-9} \mathrm{~m}^{2} \mathrm{~s}^{-1}$ [cf. Eq. (20) with $\eta=5.31 \times 10^{-5} \mathrm{~Pa} \mathrm{~s}$ the shear viscosity of Ar at $323 \mathrm{~K}$ and 630 bars [14] ]. This large difference is most likely due to the high concentration of neopentane in the sample (cf. Table I). To show this we note that for colloidal suspensions at high concentrations one finds [19]

$$
D_{s}=\frac{D_{\mathrm{SE}}}{\chi}
$$

Here $D_{\mathrm{SE}}$ is the Stokes-Einstein diffusion coefficient given by Eq. (20) and $\chi=g(\sigma)=\left(1-0.5 \phi_{\nu}\right) /\left(1-\phi_{\nu}\right)^{3}$ the pair correlation function of the larger particles at contact, with $\phi_{\nu}=\pi n \sigma^{3} / 6$ the volume fraction [15].

Applying Eq. (22) to sample III and taking the reduced density $n^{*}=n_{\text {neo }}^{*}=n_{\text {neo }} \sigma_{\text {nep }}^{3}=0.5$, we find $\chi=2.1 \pm 0.5$ so that $D_{s}=(8 \pm 2) \times 10^{-9} \mathrm{~m}^{2} \mathrm{~s}^{-1}$, in good agreement with the experimental value $D_{s}=(9 \pm 2) \times 10^{-9} \mathrm{~m}^{2} \mathrm{~s}^{-1}$. This implies that the relation Eq. (22) observed for colloids might well be valid also for binary mixtures of particles with comparable size.

\section{Rotational dynamics}

We find that for $|\omega|<1.4 \mathrm{ps}^{-1}$ the rotational dynamics of neopentane in $\mathrm{Ar}$ at $323 \mathrm{~K}$ can be described by rotational diffusion with diffusion coefficient $D_{r}=0.8 \pm 0.3 \mathrm{ps}^{-1}$. We compare this result with the rotation diffusion coefficient $D_{r}=0.4 \mathrm{ps}^{-1}$ found in pure liquid neopentane at $265 \mathrm{~K}$ [20]. Although our result is not very accurate, it seems that in the pure fluid the rotational motion is somewhat slower than in the neopentane Ar mixture. This may be caused by the difference in temperature ( 265 vs $323 \mathrm{~K}$ ) and the higher (reduced) density $n_{\text {neo }}^{*}=0.9$ of neopentane in the pure fluid (cf. $n_{\text {neo }}^{*}=0.5$ in the mixture).

We also estimate the rotational diffusion coefficient of neopentane in Ar with the continuum description of the rotation diffusion coefficient of a sphere (diameter $\sigma$ ) in a continuum with viscosity $\eta$, yielding $D_{r}=k_{B} T / \pi \eta \sigma^{3}=0.15$ $\mathrm{ps}^{-1}$, with $\eta$ the shear viscosity of Ar at 630 bars and $323 \mathrm{~K}$ [21]. Apparently, the continuum description does not hold for the rotational motions of neopentane molecules in argon. The reason for this is unclear to us.

\section{ACKNOWLEDGMENTS}

We acknowledge financial support from the Netherlands Organization for Scientific Research (NWO), and J. M. Kincaid for his contribution to the theoretical calculations.
[1] H. van Beijeren and M. H. Ernst, Physica 68, 437 (1973); 70, 225 (1973); J. Stat. Phys. 21, 125 (1979).

[2] Umberto Balucani and Marco Zoppi, Dynamics of the Liquid State (Clarendon, Oxford, 1994), Chap. 4.

[3] P. Westerhuijs, L. A. de Graaf, and I. M. de Schepper, Phys. Rev. E 48, 1948 (1993).

[4] H. E. Smorenburg, L. A. de Graaf, and I. M. de Schepper, Phys. Lett. A 181, 321 (1993).

[5] J. R. Dorfman and H. van Beijeren, in Statistical Mechanics B, edited by B. J. Berne (Plenum, New York, 1977).

[6] H. E. Smorenburg, R. M. Crevecoeur, and I. M. de Schepper, Phys. Lett. A 211, 118 (1996).

[7] H. E. Smorenburg, R. M. Crevecoeur, I. M. de Schepper, and L. A. de Graaf, Phys. Rev. E 52, 2742 (1995).

[8] S. D. Hamann and J. A. Lambert, Aust. J. Chem. 7, 1 (1954).

[9] B. L. Rogers and J. M. Prausnitz, J. Chem. Thermodyn. 3, 211 (1971).

[10] V. F. Sears, Can. J. Phys. 44, 1279 (1966); 44, 1299 (1966); 45, 237 (1967).

[11] P. Verkerk and A. M. M. Pruisken, Nucl. Instrum. Methods
160, 439 (1979).

[12] C. J. Carlile and M. A. Adams, Physica B 182, 431 (1992).

[13] V. F. Sears, Neutron News 3, 29 (1992).

[14] CRC Handbook of Chemistry and Physics, edited by R. C. Weast, 62nd ed. (CRC, Boca Raton, FL, 1982).

[15] D. A. McQuarrie, Statistical Mechanics (Harper and Row, New York, 1976).

[16] W. Montfrooij, P. Verkerk, and I. M. de Schepper, Phys. Rev. A 33, 540 (1986).

[17] J. A. A. Wouters, Delft University of Technology IRI Report No. 132-8808, 1988 (unpublished); P. Verkerk (private communication).

[18] H. M. Fijnaut, C. Pathmamanoharan, E. A. Nieuwenhuis, and A. Vrij, Chem. Phys. Lett. 59, 351 (1978).

[19] E. G. D. Cohen and I. M. de Schepper, Phys. Rev. Lett. 75, 2252 (1995).

[20] T. Mansson, L. G. Olsson, and K. E. Larsson, J. Chem. Phys. 66, 5817 (1977).

[21] R. B. Bird, R. C. Amstrong, and O. Hassager, Dynamics of Polymeric Liquids (Wiley, New York, 1987), Vol. 1. 